\title{
Micromachined optical fiber current sensor
}

\author{
R. L. Heredero, Ramón Fernández de Caleya, Héctor Guerrero, Pere Los Santos, \\ Mari Cruz Acero, and Jaume Esteve
}

\begin{abstract}
We describe a micromachined optical fiber current sensor. The sensing element consists of a squared silicon membrane $(8 \mathrm{~mm}$ long and $20 \mu \mathrm{m}$ thick) that has a cylindrical permanent magnet ( $\mathrm{NdFeB}$ alloy, 3-mm diameter, $1.5 \mathrm{~mm}$ high) fixed on its central region. This structure allows the permanent magnet to vibrate in the presence of the magnetic field gradient generated by an ac. A linear relation between the electrical current and the magnet displacement was measured with white-light interferometry with an optical fiber low-finesse Fabry-Perot microcavity. A measurement range of 0-70 A and a minimum detectable intensity of $20 \mathrm{~mA}$ were obtained when distance $D$ between the membrane and the electrical power line was $5 \mathrm{~mm}$. The output signal directly shows a linear response with distance $D$. (C) 1999 Optical Society of America
\end{abstract}

OCIS codes: $\quad 060.2370,120.3180$.

\section{Introduction}

Optical current sensors (OCS) have been investigated since the 1960's, ${ }^{1-3}$ and the benefits of optical current measurement in power systems have been widely discussed. ${ }^{4}$ Most OCS's are based on magneto-optical principles. Although Kerr effect was sometimes used as a transducing mechanism, ${ }^{5}$ systems employing Faraday effect have been the most widely developed, with both optical fibers ${ }^{6,7}$ and bulk glass materials. ${ }^{8}$ According to our knowledge, there have been published more than 600 papers and proceedings about OCS's. Several reviews give an idea of the magneto-optical and non-magneto-optical OCS. ${ }^{9,10}$

Among the non-magneto-optical devices, several magnetomechanical solutions have been suggested. Their working principle is based on the movement of an element due to the action of the magnetic field.

R. López-Heredero (lopezhr@inta.es), R. Fernández de Caleya, and H. Guerrero are with Laboratorio de Instrumentación Espacial (LINES), División de Ciencias del Espacio, Instituto Nacional de Técnica Aeroespacial (INTA), 28850 Torrejón de Ardoz, Madrid, Spain. R. Fernández de Caleya is also with the Departamento Electrónica y Sistemas, Universidad Privada de Madrid. P. Los Santos, M. C. Acero, and J. Esteve are with Centro Nacional de Microelectrónica, CNM-IMB, Consejo Superior de Investigaciones Científicas, Campus de la Universidad Autónoma de Barcelona, 08193 Bellaterra, Barcelona, Spain.

Received 11 March 1999; revised manuscript received 1 June 1999.

0003-6935/99/255298-08\$15.00/0

(C) 1999 Optical Society of America
In this sense, the cantilever principle has been exploited in intensity optical fiber sensors employing coated fibers (with ferromagnetic ${ }^{11}$ and aluminium ${ }^{12}$ jackets). Other solutions use optical fiber interferometric techniques with a piezoelectric strip to which either a Fabry-Perot fiber interferometer ${ }^{13}$ or a magnetostrictive amorph metal ring is bonded. ${ }^{14}$

In this paper, we present an OCS based on the vibration of a permanent magnet attached to the center of a silicon micromachined membrane. ${ }^{15}$ The magnet moves as a consequence of a time-dependent magnetic field gradient generated by an electric power line. This transducing scheme has been reported for a magnetometer in which the membrane deflection is capacitively measured, ${ }^{16}$ and therefore this technique is not suitable to be implemented in high-voltage applications.

We detect membrane deflection with white-light interferometry (WLI) with an optical fiber low-finesse Fabry-Perot (LFFP) microcavity. Micromachined Si structure for pressure sensors has been previously implemented with WLI technique. ${ }^{17}$ The LFFP interferometer of our OCS is formed between the end of an optical fiber and a mirror fixed on the magnet. As we show, the presented OCS matches a linear transduction mechanism with a completely optical fiber detection system.

The sensor presented here uses a standard singlemode optical fiber and has no limitation about the LED central wavelength. There is a great advantage in feasibility and price, because the commonly used LED's, detectors, and single-mode optical fibers 


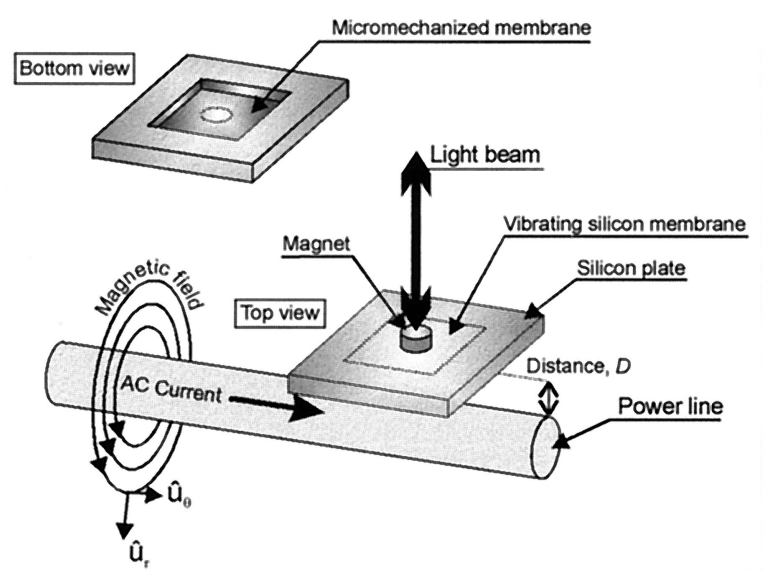

Fig. 1. Micromachined Si transducer working principle. An ac generates a magnetic field gradient that produces a permanent magnet to vibrate on a Si membrane. This movement is detected optically.

used in optical communications can be employed in the manufacturing of the sensor.

Another singularity is that the transducing mechanism is based on a micromachined Si structure. This material is widely used in sensors, actuators, and microelectromechanical systems (MEMS). The Si technology is widespread and well known, and its mechanical and thermal behavior can be predictable.

\section{Micromachined Transducer: Design and Operation Principle}

\section{A. Operation Principle}

The presented OCS is composed of a transducing head (Fig. 1) and an optical fiber interferometric detection system. The transducer consists of a cylindrical $\mathrm{NdFeB}$ permanent magnet $\left(B_{r}=1.1 \mathrm{~T}, 3-\mathrm{mm}\right.$ diameter, and 1.5-mm high) fixed on the central region of a squared Si membrane $(8 \mathrm{~mm}$ long and $20 \mu \mathrm{m}$ thick). The actuator design is a simple one-mask process that defines the zones of the wafer to be removed. This process begins with a (100) oriented wafer. An oxide layer protects both sides of the wafer while tetramethyl ammonium hydroxide (TMAH) etching anisotropically removes the silicon from the bulk where the oxide has been etched after a wellestablished etching time ratio. The thickness resolution of this process is nearly $2.5 \mu \mathrm{m}$, and a minimum membrane thickness of $10 \mu \mathrm{m}$ can be achieved. In this case the membrane is $20 \mu \mathrm{m}$ thick, and the silicon wafer used has a thickness of $325 \mu \mathrm{m}$ and a diameter of 4 in. $(10.16 \mathrm{~cm})$.

If this structure is located at a certain distance $D$ from an electrical power line carrying an electric intensity $I_{\text {elec }}$, there will be a Lorentz force $\mathbf{F}$ on the magnet. This force can be expressed by

$$
\mathbf{F}=M V_{m} \nabla H,
$$

with $M$ and $V_{m}$ being the magnetization and the volume of the magnet, respectively, and $\nabla H$ being the magnetic field gradient associated to $I_{\text {elec }}$, at the mag- net position. If an ideal power line is considered, the magnetic field $\mathbf{H}$ generated by $I_{\text {elec }}$ at a distance $D$ can be expressed in cylindrical coordinates as follows:

$$
\mathbf{H}=\frac{\mathbf{B}}{\mu}=\frac{I_{\text {elec }}}{2 \pi D} \hat{\mathbf{u}}_{\theta} .
$$

From this expression $\nabla H$ can be easily obtained:

$$
\nabla H=\frac{I_{\text {elec }}}{2 \pi D^{2}} \hat{\mathbf{u}}_{r}
$$

In Eq. (1) it is shown that the force acting on the magnet points in the direction of $\nabla H$, that is, along as the radial direction.

From the point of view of the Si membrane, a force acting on it causes a deflection. The magnitude and direction of such displacement depends on the vibration conditions of the membrane and on the relative position between the power line and the transducer. In any case, it is assumed that the magnet, under the action of a force $\mathbf{F}$, is shifted a magnitude $\Delta l$ from its equilibrium position. For small displacements, we can establish that

$$
|\mathbf{F}|=K \Delta l,
$$

with $K$ being a frequency-dependent coefficient related to the Si membrane structure and the elasticity of this material.

If the transducer is placed on a plane parallel to the power line, then by substituting Eq. (3) into Eq. (1) and by considering Eq. (4) it can be demonstrated that the electrical current $I_{\text {elec }}$ is proportional to the displacement produced in the transducer:

$$
I_{\text {elec }}=\left(\frac{2 \pi D^{2} K}{M V_{m}}\right) \Delta l .
$$

Equation (5) indicates that there is a linear relation between the electrical current $I_{\text {elec }}$ and the deflection of the membrane $\Delta l, I_{\text {elec }} \propto \Delta l$. From the point of view of the OCS, this linearity is important because it simplifies the subsequent signal treatment. We show that the sensor exhibits also a linear response with the distance $D$.

The membrane deflection (in approximate micrometers) can be measured by means of interferometric techniques; the phase change of the light beam reflected on the surface of the magnet gives information about the membrane displacement. For this purpose, the surface of the magnet has been covered with a Si plate that acts as a mirror. Furthermore, optical fringes can be achieved by use of optical fibers where transducers can be easily implemented. These fiber architectures are strongly recommended, especially for noisy environments such as highvoltage power lines.

\section{B. Optical Characterization}

For a long time, interferometric techniques have been widely used for highly sensitive measurements of a variety of physical parameters. If a two-beam inter- 


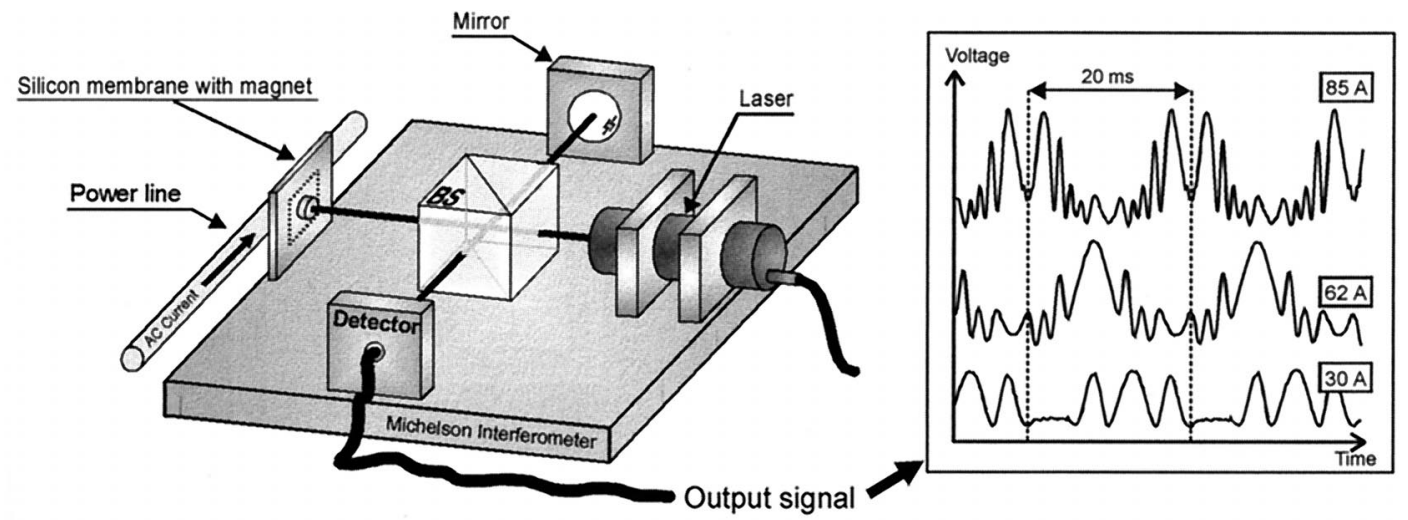

Fig. 2. Michelson bulk configuration. The graph on the right shows detected signals for $I_{\text {elec }}$ equal to 30,62 , and $85 \mathrm{~A}$ when the $\mathrm{Si}$ membrane was allocated at $D=3 \mathrm{~mm}$ from the power line.

ferometer is considered, the detected intensity of the signal at the receiver, $I_{\text {det }}$, has the well-known equation 18 :

$$
I_{\text {det }}=I_{0}(1+V \cos \phi),
$$

where $I_{0}$ is the continuous level of the detected intensity, $V$ is the visibility of the signal, and $\phi$ describes the optical phase difference between the two interferometric paths.

In the presented OCS the optical phase of the light $\phi$ is modulated by the time dependence of the magnetic field generated by the electric current. Since the power line works at 50 or $60 \mathrm{~Hz}$ (in our case, $f_{s}=$ $50 \mathrm{~Hz}$ ), the force that acts on the magnet induces a time-dependent phase shift with a periodicity of $1 / f_{s}$ owing to the sinusoidal variation of the length of the sensing branch. This differential phase shift may be separated into an information term of the electrical current of amplitude $\phi_{s}$, which oscillates at frequency $f_{s}$, and a slowly varying phase drift $\phi_{d}$, which is produced by the total phase noise of the interferometer. Therefore the resulting time-dependent optical phase can be expressed as

$$
\phi(t)=\phi_{d}+\phi_{s} \cos \left(2 \pi f_{s} t\right) .
$$

The amplitude of the phase shift, calculated from Eq. (5), takes the following form:

$$
\phi_{s}=\frac{2 \pi n}{\lambda} 2, \quad \Delta l=\frac{2 n M V_{m}}{K D^{2} \lambda} I_{\text {elec }},
$$

where $n$ is the refractive index of the interferometer interaction zone, $\lambda$ is the wavelength of the light source, and $\Delta l$ is the deflection of the membrane.

To prevent undesired fluctuations of $\phi_{d}$ in our experimental setup, we used a bandpass filter centered at $f_{s}$ and a lock-in amplifier. However, other interrogation techniques could be also considered to extract the information from the optical phase free of such noise.

The micromachined Si transducer was first tested in a Michelson bulk configuration, as is shown in Fig. 2. The transducer was placed at the end of the in- terferometer sensing branch and acted as a movable reflector. The optical source used was a He-Ne laser $(632.8 \mathrm{~nm})$, and a visibility greater than $15 \%$ was obtained for a minimum optical path difference (OPD) between the two branches of the interferometer.

According to Eq. (7), for the intensity range considered $(0-70 \mathrm{~A})$, the peak-to-peak variation of $\phi(t)$ was large enough to push the sensor phase signal $\phi_{s}$ higher than $\pi / 2$. Hence we performed the calibration of the transducer by counting interference fringes (each fringe representing a $\pi / 2$ phase shift) for a given distance $D$.

In Fig. 2, detected signals are reported for $I_{\text {elec }}$ equal to 30,62 , and $85 \mathrm{~A}$ and $f_{s}=50 \mathrm{~Hz}$, when the transducer was allocated at $D=3 \mathrm{~mm}$ from the power line. Notice the increment of the number of fringes when $I_{\text {elec }}$ increases. In these curves, an overintensity modulation centered at $50 \mathrm{~Hz}$ can be appreciated when large deflections are caused on the membrane. This effect indicates that optical intensity modulation starts to be appreciable and overlaps to the signal produced by the phase optical fringes. If larger deflections are produced, a pure intensitymodulation sensor is achieved and optical fringes disappear. We have observed this effect for extremely large deflections $(>0.3 \mathrm{~mm})$ obtained at the resonance frequency of the membrane. This structure responds to a current range from 0 to $190 \mathrm{~A}$. This data was achieved for distances between 5 and 13 $\mathrm{mm}$.

Results in Fig. 3 were obtained for various distances $D$. As it can be expected from Eq. (8), the relation between number of fringes and current intensity is linear. The resolution of the measurement was \pm 1 fringe $( \pm \lambda / 8)$. Measurements in Fig. 3 were made five times for each value of $D$, and the relation between slopes of the curves in Fig. 3 and distance $D$ was determined; results are represented in Fig. 4. The fit corresponds to a $D^{-2}$ dependence, as it could be expected from Eq. (8). Current measurements in high-power delivery systems are in the range of several thousands amperes. If we wanted to measure 


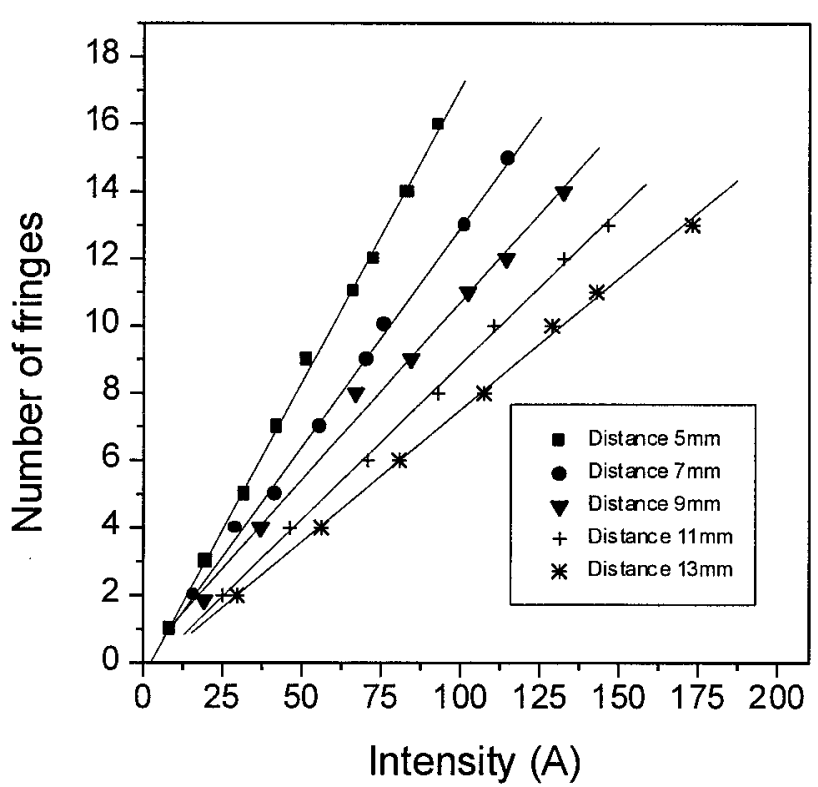

Fig. 3. Response of the current probe measured for various distance separations $(D)$ between the power line and the Si membrane. Relation between number of fringes and intensity is linear. The slopes of these lines are $0.17,0.12,0.11,0.09$, and 0.08 (fringes/A) for distances $D=5,7,9,11$, and 13 (mm), respectively.

such high currents with a smooth signal profile, we only need to increase the distance $D$.

One important aspect to consider is the frequency dependence of the deflection of the Si membrane. We measured it interferometrically and show the results in Fig. 5. We performed this test by exciting the membrane with a 0.4-A electrical current applied

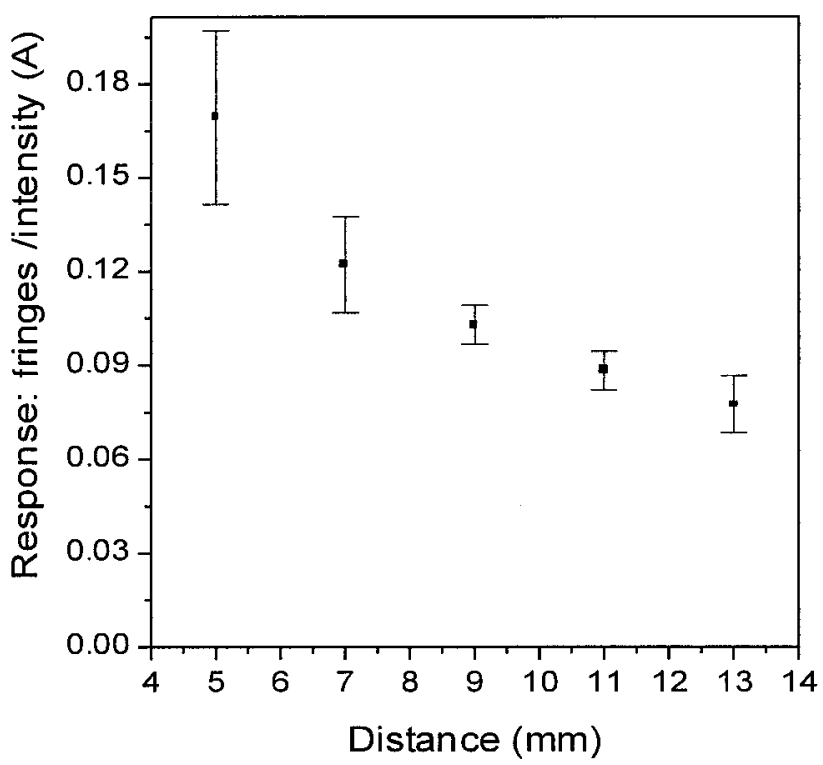

Fig. 4. Relation between slopes of response of the sensor (fringes versus intensity) against distance $D$. Data were taken five times; points show arithmetic mean value of experimental data, and vertical error bars show standard deviation. The polynomial fit shows a $D^{-2}$ dependence, as could be expected from Eq. (8).

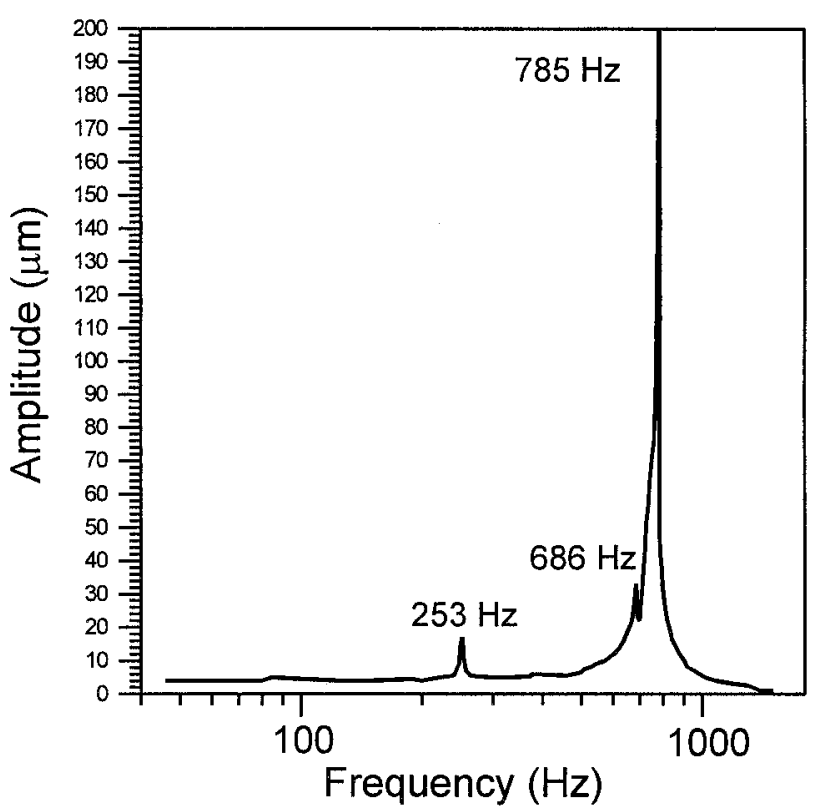

Fig. 5. Frequency response of the Si membrane. Ordinates axis shows displacement of the membrane, $d$ described by $\phi=(4 \pi /$ $\lambda) n d$, where $\phi$ is the phase of the detected signal $I_{\text {det }}$. Measurement was made by counting interference fringes ( 1 fringe corresponds to $\pi / 2 \mathrm{rad}$ ).

on a planar rectangular coil (10 turns). Far from the main membrane resonance at $f r=785 \mathrm{~Hz}$ or from the secondary peak at $253 \mathrm{~Hz}$, the displacement amplitude remains nearly constant for the rest of the spectrum. Since the resonance frequency $f_{r}$ changes with temperature, the sensor should work at frequen$\operatorname{cies} f_{s}$ far enough from $f_{r}$. This is verified for the usual frequencies of 50 and $60 \mathrm{~Hz}$.

Note that most of the Si-membrane sensors referenced above are limited to work at the membrane resonance frequency if a maximum response of a directly intensity-modulated sensor is to be obtained. However, we are considering a phase-modulated sensor and therefore, higher sensitivity is achieved. In this case, the response in a nonresonance spectral region is enough for our sensing requirements. Besides, working at the resonance frequency implies nonlinearity in the transducing function and extreme dependence on sensor external conditions. In fact, the resonance frequency shift of vibrating structures is used as a common transducing principle.

\section{Optical Fiber Current Sensor}

\section{A. Experimental Setup}

In many applications, small sensing heads are needed to perform point measurements. In these cases, a LFFP interferometer (also known as Fizeau interferometer) with a cavity length of approximately several micrometers is an attractive choice for the sensor element. ${ }^{19,20}$ A problem arises with LFFP when its cavity length exceeds the coherence length of the light source: no interference occurs at the output of the LFFP. In this case, a low-coherence (or 


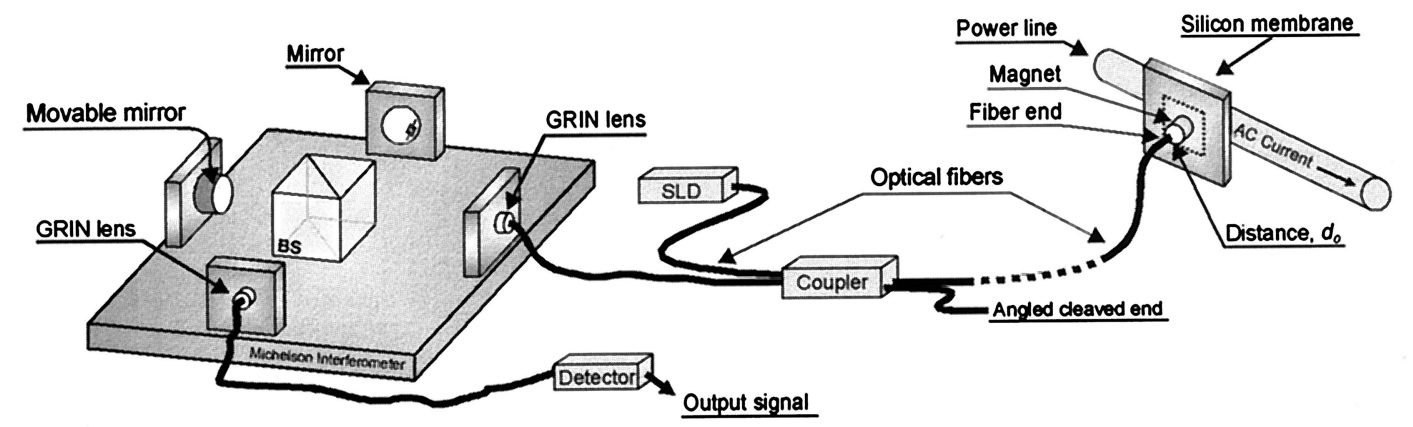

Fig. 6. Setup of the WLI system. Sensing interferometer is a LFFP microcavity, and the receiving interferometer is a Michelson bulk configuration.

white-light) technique is used to recover the induced phase changes in the signal interferometer to be measured. This can be achieved having the LFFP cavity operating in conjunction with a receiving interferometer.

For the micromachined transducer shown in Fig. 1 to have an operative sensor, it was located at the end of an optical fiber forming a LFFP microcavity that had been integrated into a remote sensing system based on WLI. The receiving interferometer was a Michelson bulk. The experimental setup of the WLI is shown schematically in Fig. 6 .

The LFFP is formed between the perpendicular cleaved end of the single-mode fiber and the plate of Si mounted onto the magnet. Since the Fresnel reflection from the glass-optical-fiber-air interface is small, the multiple beams received from the LFFP can be approximated to a signal of a two-beam interferometer. ${ }^{21}$ Therefore it can be assumed that the two main terms of the detected intensity at the output of the WLI system can be expressed by Eq. (6) with the sign + being modified to a - because of the reflective Airy function.

The path-matching condition, necessary for whitelight interference effects to be observed at the output of the receiving interferometer, requires that the difference between the length of the cavity and the OPD of the Michelson bulk falls within the coherence length of the light source of the system. The zeroorder fringe corresponds to the exact optical-path match of the two beams and has the highest amplitude. The coherence tuning of the receiving interferometer is always advantageous because it allows for the sensing interferometer to remain entirely passive.

\section{B. Results}

The light source used in this research was a pigtailed superluminescent diode (SLD) $(\lambda=833 \mathrm{~nm})$. A single-mode fiber $\left(\lambda_{c}=727 \mathrm{~nm}\right)$ is used as the inputoutput fiber, and the beam is injected to a $2 \times 2,3-\mathrm{dB}$ directional coupler. The free fiber branch at the output of the coupler was cleaved with a Brewster angle to avoid unwanted reflections from this path. The photodetector used is a Hewlett-Packard Lightwave Multimeter (Model HP 8153A) with a power sensor
(Model 81530A). The average optical power received at the detector was greater than $1 \mathrm{nW}$. The static length of the cavity $d_{0}$ was larger $(\sim 50 \mu \mathrm{m})$ than the coherence length of the SLD used $(l c \sim 30$ $\mu \mathrm{m}, \lambda=833 \mathrm{~nm}$ with $\Delta \lambda=23.2 \mathrm{~nm}$ ). Once the maximum central fringe is detected, the visibility of the signal was $\sim 17 \%$.

The optical phase detected at the output of the Michelson bulk interferometer was always less than $\pi / 2$ for a measured intensity range from 0 to $70 \mathrm{~A}$. Thus calibration of the sensor head was achieved by measurement of the rms voltage detected at the output of the detector $\left(V_{\mathrm{rms}}\right)$. The response of the sensor is displayed in the three plots contained in Fig. 7. Figure 7(a) shows the typical sensor output for several currents. $V_{\text {rms }}$ was measured for several currents $I_{\text {elec }}$ and distances $D$. Results are displayed in Fig. 7(b). There is a cosine dependency between the $V_{\text {rms }}$ and the electric intensity current. After an inverse cosine demodulation, the results showing a linear relation with intensity are displayed in Fig. 7(c).

We have also investigated the sensitivity of the OCS for a distance $D \sim 4.45 \mathrm{~mm}$. The interferometric minimum phase resolution measured was $\Delta \phi_{\min }=640 \mu \mathrm{rad} / \sqrt{ } \mathrm{Hz}$ at $50 \mathrm{~Hz}$, which corresponds to a minimum detectable intensity of $26 \mu \mathrm{A} / \sqrt{ } \mathrm{Hz}$. A dynamic range of $47.7 \mathrm{~dB}$ results for the OCS for electric currents between 0 and $70 \mathrm{~A}$.

A final test performed on the OCS was the measurement of the rms voltage variation with distance $D$. Values measured for an ac of $20 \mathrm{~A}$ are shown in Fig. 8. Because of the linear dependency exhibited one can envisaged an easy implementation of several OCS's (i.e., 3) on a ring structure and in such a way that a sensor insensitive to the power line position will be achieved by triangulation.

\section{Discussion}

An optical fiber current sensor has been developed with a micromachined Si membrane. This sensor verifies the main requirements to be employed in high-voltage power lines: it is remotely monitorized by an optical fiber (dielectric medium) and is insensitive to electromagnetic interferences. Another advantage of the presented OCS is that there are no limitations about the emitter wavelength, thus low- 
(a)
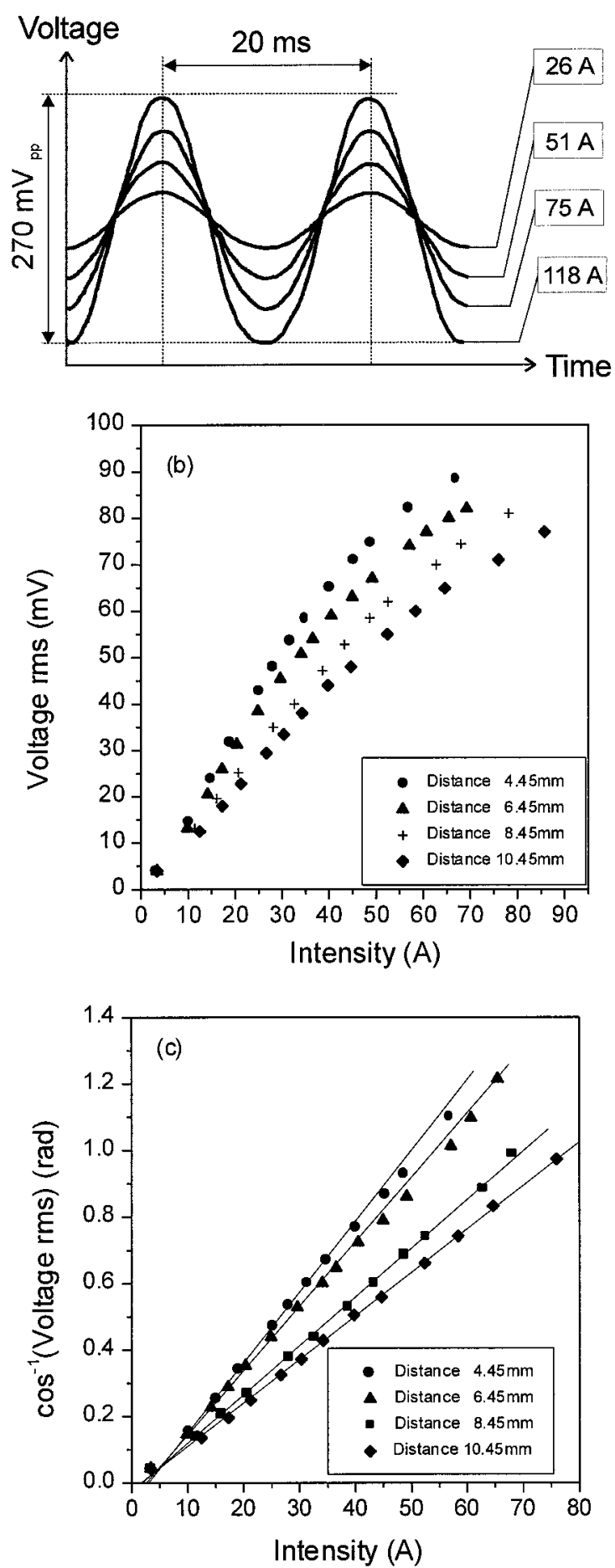

Fig. 7. (a) Typical sensor output for several intensity currents $\left(I_{\text {elec }}\right)$ when $D \sim 2.5 \mathrm{~mm}$. When $I_{\text {elec }}$ was $26,51,75$, and $118 \mathrm{~A}$, $V_{\text {rms }}$ was $20.1,41.8,60.4$, and $90.9 \mathrm{mV}$, respectively. (b) $V_{\text {rms }}$ against $I_{\text {elec }}$ for various distances $D$. These curves show a cosine dependency. (c) Inverse cosine demodulation of the $V_{\text {rms }}$ of Fig. 7(b). The lines represented are the linear fits.

coherence sources such as LED's can be used. Therefore, all the standard technology from telecommunications (emitters, detectors, and optical fibers)

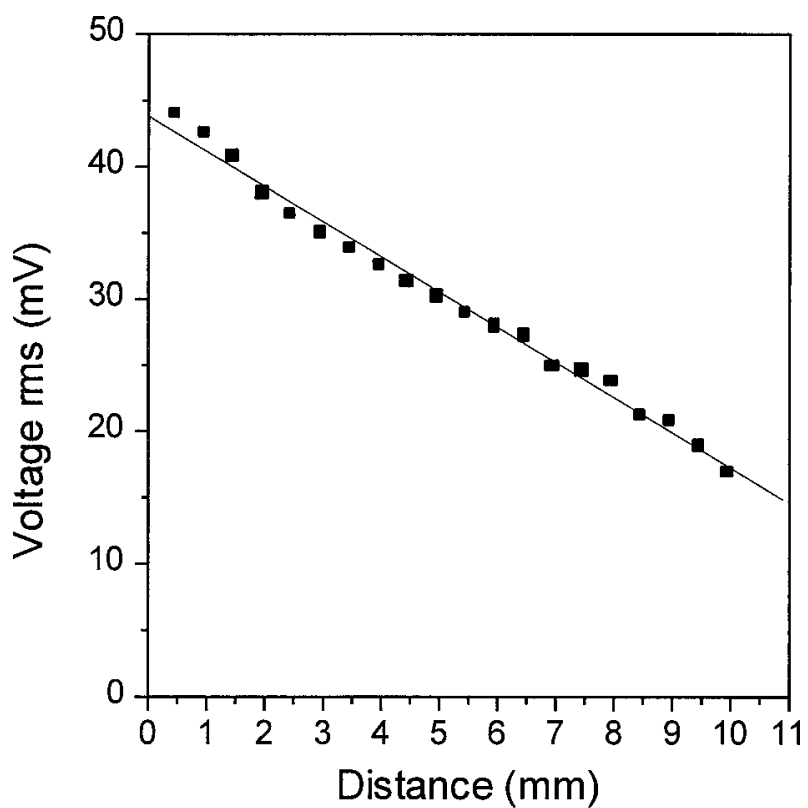

Fig. 8. $V_{\text {rms }}$ against $D$ when the current intensity $I_{\text {elec }}$ was $\sim 19.9$ A. Notice that a linear dependency has been achieved.

is available for this OCS. We must only take care about the sensitivity, because in interferometric sensors, the lower the wavelength, the higher the sensitivity.

This OCS could be considered as a magnetic field sensor. However, its working principle is based on the action of the magnetic field gradient, causing the displacement of a magnet. A homogeneous magnetic field would not be detected, and therefore this transducer cannot be treated as a true magnetic field sensor. Our OCS is intended to detect electrical currents owing to the intrinsic radial distribution of the magnetic field generated by the power line. The effect of other surrounding lines can be minimized by deployment of the OCS in such a way that the normal direction to the $\mathrm{Si}$ membrane is parallel to the $u_{\theta}$ coordinate (Fig. 1).

Experimental results and theoretical calculations presented here show that the characterization and the sensor response exhibit a linear and predictable behavior. The detection scheme WLI is well known, and there is a lot of research developed in this field. The electrical intensity can be directly extracted from the inverse cosine of the output voltage of the receiving interferometer in the WLI scheme. As we have seen in Fig. 8, this cosine relation partially compensates the $D^{-2}$ dependency of the slope of the curves as is represented in Figs. 3 and 4, and this yields a quasi-linear response of the sensor with $D$.

From the point of view of real field installation of an OCS, which requires high demands on reliability and accuracy over a long time, other factors must be taken into account: immunity to vibration and temperature stability over a broad range (typically, from $-20{ }^{\circ} \mathrm{C}$ to $+80^{\circ} \mathrm{C}$ ). Our OCS does not work on the basis of polarization principles (i.e., as Faraday ef- 
fect), and therefore it is unaffected by optical noise due to polarization induced by fiber-link vibration ${ }^{22}$ or by mechanical vibrations that induce unwanted birefringence changes in OCS. ${ }^{23,24}$ In this sense, the use of a standard single-mode optical fiber against special polarization optical fibers, such as low birefringence, is a clear advantage in price and in implementation.

Temperature is the main drawback for most OCS's. In Faraday-effect sensors, the trends are to use diamagnetic media. ${ }^{8}$ In general, it can be established that the Verdet constants of diamagnetic glasses are nearly independent of temperature, ${ }^{25}$ but, in fact, temperature drifts affects them. ${ }^{26}$ A major effect is the change induced by temperature in the residual birefringence of an OCS based on Faraday effect. For temperature compensation, some solutions, such as the use of a temperature probe in the sensor head ${ }^{27}$ or with methods based on signal processing, ${ }^{28}$ have been presented. The temperature dependence of our OCS is under investigation. Temperature can affect the transducer by its changing the magnet magnetization $M$ (typically $\sim 0.04 \% /{ }^{\circ} \mathrm{C}$ for $\mathrm{SmCo}$ magnets), the Young's modulus, or the longitudinal elasticity of the $\mathrm{Si}$, the $E$, and the dynamical conditions of the membrane. Variations of $M$ and $E$ with temperature higher than a $100-^{\circ} \mathrm{C}$ range are minor and work in opposite direction. Therefore, these two variables do not considerably affect the thermal behavior of the OCS. However, we have measured that a small temperature drift can strongly affect the output of the OCS if the membrane is not clamped correctly. The membrane is surrounded by a $\mathrm{Si}$ frame, which must be mounted on a structure that allows it to undergo free thermal expansion. If the membrane frame is fixed on a rigid support, a thermal expansion of the frame is produced toward the center of the membrane, causing some changes in its dynamical conditions. This generates a mechanical stress that affects the transducer response. We have measured a thermal dependency of $+2 \% /{ }^{\circ} \mathrm{C}$ in the interval from 20 to $50{ }^{\circ} \mathrm{C}$ from the slopes of the fits represented in Fig. 3. This behavior could be typically of a temperature transducer. For solution of this temperature dependency, a mechanical support specially designed to allow free expansion of the membrane is under development. Because of the possibility of mechanizing the Si transducer, future development of micromachined structures for temperature compensation can be also envisaged.

\section{Conclusions}

An optical fiber current sensor based on a permanent magnet fixed on the center of a Si membrane has been presented. The transducing operation principle is magnetomechanical and has a linear response. This behavior has been optically tested at frequencies as high and higher than $1 \mathrm{kHz}$ and for amplitudes below $10 \mu \mathrm{m}$. Optical interrogation of the transducer with a WLI arrangement has been used in the development of an OCS. Its response can be extracted by means of an inverse cosine demodulation yielding a linear function with current. A measurement range of 0-70 A and a minimum detectable intensity of 20 $\mathrm{mA}$ (sensitivity of $\sim 3 \times 10^{-4}$ ) were obtained when distance $D$ between the membrane and the electrical power line was $5 \mathrm{~mm}$.

We have also measured the linear behavior of the OCS output with the distance from the sensor head to the power line. This will be exploited for development of a system insensitive to power line position. This system can be achieved with several OCS's mounted on a ring structure, and the final signal will be obtained by a triangulation method. This configuration will overcome problems related to remanent birefringence, which affects the typical ring-type structures used for verifying Ampere's Law with Faraday-effect materials.

The substantial advantage that our OCS presents over other traditional methods for measuring current relies on the fact that the transducing principle can be exploited with standard low-cost telecommunication components (standard single-mode optical fibers and low-coherence emitters) without the utilization of polarizing components. The working principle of our OCS is not linked to any special wavelength range, unlike the Faraday-effect sensors in which the Verdet constant depends strongly on wavelength. Finally, the interferometric detection scheme takes advantage of the natural frequency of the current in the power line $(50$ or $60 \mathrm{~Hz}$ ), which implies inherent reduction of low-frequency noise.

At present, the thermal behavior of the sensor is being studied. For temperature dependency of the OCS to be overcome, a new fixing grip for the membrane is under development. In this sense, a broad spectrum of improvements is envisaged owing to micromachined possibilities of Si. This will allow for the manufacturing of new bending surfaces and new structures for interfacing the optical fiber to the vibrating membrane, which will make it easier to implement in a real sensor.

The authors thank to J. Rodriguez and to J. Ruiz of Departamento de Ciencia de Materiales, Escuela Técnica Superior de Ingenieros Caminos Canales y $\mathrm{Pu}-$ ertos of Universidad Politécnica de Madrid for helpful discussion about the thermal and the mechanical aspects of the Si membrane and its frame.

This research has been partially supported by Comisión Interministerial de Ciencia y Tecnología, Project TIC95-0631-C04-03.

\section{References}

1. S. Saito, Y. Fujii, K. Yokoyama, J. Hamasaki, and Y. Ohno, "The laser current transformer for EHV power transmission lines," IEEE J. Quantum Electron. 2, 255-259 (1966).

2. Y. Pelenc, "Transoptics: current transformers using magnetooptic effect for very high voltages," L'Electricien 82, 63-66 (1969).

3. W. Hermstein, "Development trends in the construction of measuring transformers-with special reference to unconventional instrument transformers for high voltages," Elektrizitaetswirtschaft 68, 246-257 (1969).

4. Emerging Technologies Working Group, Power Systems In- 
strumentation and Measurements Committee, the Fiber Optic Subcommittee, Power Systems Communications Committee, "Optical current sensors for power systems: a review," IEEE Trans. Power Deliv. 9, 1778-1788 (1994).

5. S. Matsushita, S. Konishi, and Y. Sakurai, "Magnetooptical ammeter," IEEE Trans. Magn. 6, 569-571 (1970).

6. A. D. Higham and J. D. Newman, "Measurement of current using Faraday rotation in glass fiber," in Colloquium Digest on Novel Types of Transducer (IEEE, London, UK), pp. 1-2.

7. G. W. Day and A. H. Rose, "Faraday effect sensors: the state of the art," in Fiber Optic and Laser Sensors VI, R. P. DePaula and E. Udd, eds., Proc. SPIE 985, 138-150 (1988).

8. Y. N. Ning and D. A. Jackson, "Review of optical current sensors using bulk-glass sensing elements," Sens. Actuators A 39, 219-224 (1993).

9. Y. N. Ning, Z. P. Wang, A. W. Palmer, K. T. V. Grattam, and D. A. Jackson, "Recent progress in optical current sensing techniques," Rev. Sci. Instrum. 66, 3097-3111 (1995).

10. Z. P. Wang, S. Q. Zhang, and L. B. Zhang, "Recent advances in optical current-sensing techniques," Sens. Actuators A 50, 169-175 (1995).

11. E. F. Carome, V. E. Kubulins, R. L. Flanagan, and P. ShamrayBertaud, "Intensity-type fiber optic electric current sensor," in Fiber Optic and Laser Sensors XI, R. DePaula and E. Udd, eds., Proc. SPIE 1584, 110-117 (1991).

12. P. D. Dinev, "A two-dimensional remote fiber-optic magnetic field and current sensor," Meas. Sci. Technol. 7, 1233-1237 (1997).

13. Y. Park, W. Seo, C. E. Lee, and H. F. Taylor, "Fiber FabryPerot type optical current sensor with frequency ramped signal processing scheme," J. Opt. Soc. (Korea) 2, 74-9 (1998).

14. Y. Zhan and M. Lu, "The analysis of an all multimode fiber electric current sensor with a fiber Fabry-Perot interferometer," in International Conference on Optoelectronic Science and Engineering '90, E. M. Campbell, ed., Proc. SPIE 1230, 539540 (1990).

15. B. Wagner and W. Benecke, "Magnetically driven microactuator," in Microsystem Technologies 90 H. Reichl, ed. (SpringerVerlag Berlin, 1990), pp. 838-843.
16. F. Ayela, T. Fournier, and J. Chaussy, "A micromachined silicon magnetometer," Sens. Actuators A 61, 339-341 (1997).

17. R. A. Pinnock, "Micromachined silicon resonant sensors with robust optical interrogation," in Sensors VI: Technology Systems and Applications, K. T. V. Grattan and A. T. Augousti, eds., (Institute of Physics Publishing, Bristol, 1993), Sec. B, pp. 141-146.

18. M. Born and E. Wolf, Principles of Optics (Pergamon, Oxford, 1980), Chap. 7.

19. A. D. Kersey, D. A. Jackson, and M. Corke, "A simple fiber Fabry-Perot sensor," Opt. Commun. 45, 71-75 (1983).

20. K. A. Murphy, M. F. Gunther, A. M. Vengsarkar, and R. O. Claus, "Quadrature phase-shifted, extrinsic Fabry-Perot optical fiber sensors," Opt. Lett. 16, 273-275 (1991).

21. J. L. Santos, A. P. Leite, and D. A. Jackson, "Optical fiber sensing with a low-finesse Fabry-Perot cavity," Appl. Opt. 31, 7361-7366 (1992).

22. N. E. Fisher and D. A. Jackson, "A common-mode optical noiserejection scheme for an extrinsic Faraday current sensor," Meas. Sci. Technol. 7, 796-800 (1996).

23. N. C. Pistoni and M. Martinelli, "Vibration-insensitive fiberoptic current sensor," Opt. Lett. 18, 314-316 (1993).

24. A. J. Rogers, J. Xu, and J. Yao, "Vibration immunity for optical-fiber current measurement," J. Lightwave Technol. 13, 1371-1377 (1995)

25. M. J. Weber, "Faraday rotator materials for laser systems," in Laser and Nonlinear Optical Materials, L. G. DeShazer, ed., Proc. SPIE 681, 75-90 (1986).

26. P. A. Williams, A. H. Rose, G. W. Day, T. E. Milner, and M. N. Deeter, "Temperature dependence of the Verdet constant in several diamagnetic glasses," Appl. Opt. 30, 1176-1178 (1991).

27. T. W. Cease, J. G. Driggans, and S. J. Weikel, "Optical voltage and current sensors used in a revenue metering system," IEEE Trans. Power Deliv. 6, 1374-1379 (1991).

28. P. Menke and T. Bosselmann, "Temperature compensation in magnetooptic ac current sensors using an intelligent ac-dc signal evaluation,” J. Lightwave Technol. 13, 1362-1370 (1995). 